\title{
A COMMON FIXED POINT THEOREM FOR THE $\psi$-CONTRACTIVE MAPPING
}

\author{
CHI-MING CHEN AND TONG-HUEI CHANG
}

\begin{abstract}
In this paper, we shall discuss the common fixed point theorems of four single-valued functions with $\psi$-contractive codition in cone metric spaces.
\end{abstract}

\section{Introduction and Preliminaries}

Huang and Zhang [1] and P. Vetro [3] generalized the concept of a metric space, replacing the set of real numbers by an ordered Banach space and obtained some common fixed point theorems for mappings satisfyng some contractive conditions. In this paper, in the framework of cone metric spaces, we obtain a common fixed point theorem for four mappings that satisfies a $\psi$-contractive codition.

Let $E$ be an arbitrary real Hausdorff topological vector space, $\mathcal{P}=\{P \mid P$ is a family of seminorms which determines the topology on $E\}$, and let $C \subset E$ be a proper closed convex and pointed cone with apex at the origin and int $C \neq \phi$ (see, [2]), that is, $C$ satisfies the following conditions:

(i) $C$ is proper closed with $i n t C \neq \phi$,

(ii) $\lambda C \subset C$, for all $\lambda>0$

(iii) $C+C \subset C$, and

(iv) $C \cap(-C)=\{0\}$.

Given a proper closed convex and pointed cone $C$ with apex at the origin, we define a linearly ordering $\leq$ with respect to $C$ by $x \leq y$ if and only if $y-x \in C$. We shall write $x<y$ to indicate that $x \leq y$ but $x \neq y$, while $x<<y$ will stand for $y-x \in$ intC.

Definition 1. The cone $C$ is called normal if for each $p \in P$, there exists a number $k_{p}>0$ such that for all $x, y \in E$,

$$
0 \leq x \leq y \text { implies } p(x) \leq k_{p} p(y) .
$$

The least positive number $k_{p}$ satisfying above is called the normal constant of $C$ with repect to each seminorm $p, p \in P$.

Corresponding author: Tong-Huei Chang.

Received December 25, 2008; revised March 11, 2009.

2000 Mathematics Subject Classification. 47H10, 54C60 , 54H25, 55M20.

Key words and phrases. Cone metric space, compatible, common fixed point, $\psi$-contractive.

Research supported by the NSC. 
Definition 2. The cone $C$ is called regular if every increasing sequence which is bounded from above is convergent. That is, if $\left\{x_{n}\right\}$ is a sequence such that

$$
x_{1} \leq x_{2} \leq x_{3} \leq \cdots \leq x_{n} \leq \cdots \leq y
$$

for some $y \in E$, then there is $x \in E$ such that $\lim _{n \rightarrow \infty} p\left(x_{n}\right)=p(x)$ with repect to each seminorm $p, p \in P$. Equivalently, the cone $C$ is regular if and only if every decreasing sequence which is bounded from below is convergent.

Remark 1. It is clear that a regular cone is a normal cone.

In the following we always suppose that $E$ is a real Hausdorff topological vector space, $C$ is a proper closed convex and pointed cone in $E$ with apex at the origin and $i n t C \neq \phi$, and $\leq$ is a linearly ordering with respect to $C$.

We generalize the definition of a cone metric space [1] and P. Vetro [3], as follows:

Definition 3. Let $X$ be a nonempty set. Suppose the mapping $d: X \times X \rightarrow E$ satisfies

(i) $0<d(x, y)$, for all $x, y \in X, x \neq y$,

(ii) $d(x, y)=0$ if and only if $x=y$,

(iii) $d(x, y)=d(y, x)$, and

(iv) $d(x, y)+d(y, z) \geq d(x, z)$, for all $x, y, z \in X$.

Then $d$ is called a cone metric on $X$, and $(X, d)$ is called a cone metric space.

Definition 4. Let $(X, d)$ be a cone metric space, and let $\left\{x_{n}\right\}$ be a sequence in $X$ and $x \in X$. If for every $c \in C$ with $0<<c$, there is $N$ such that

$$
d\left(x_{n}, x\right)<<c, \text { for all } n>N,
$$

then $\left\{x_{n}\right\}$ is said to be convergent and $\left\{x_{n}\right\}$ converges to $x$.

Definition 5. Let $(X, d)$ be a cone metric space, and let $\left\{x_{n}\right\}$ be a sequence in $X$. We say that $\left\{x_{n}\right\}$ is a Cauchy sequence if for any $c \in E$ with $0<<c$, there is $N$ such that

$$
d\left(x_{n}, x_{m}\right)<<c, \text { for all } n, m>N,
$$

Definition 6. Let $(X, d)$ be a cone metric space. If every Cauchy sequence is convergent in $X$, then $X$ is called a complete cone metric space.

Follows above definitions, we are easy to show the following lemmas.

Lemma 1. Let $(X, d)$ be a cone metric space, $C$ be a normal cone with normal constant $k_{p}$ for each $p \in P$, and let $\left\{x_{n}\right\}$ be a sequence in $X$. Then $\left\{x_{n}\right\}$ converges to $x$ if and only if $d\left(x_{n}, x\right) \rightarrow 0$ as $n \rightarrow \infty$.

Lemma 2. Let $(X, d)$ be a cone metric space, and let $\left\{x_{n}\right\}$ be a sequence in $X$. If $\left\{x_{n}\right\}$ is convergent in $X$, then $\left\{x_{n}\right\}$ is a Cauchy sequence. 
Lemma 3. Let $(X, d)$ be a cone metric space, $C$ be a normal cone with normal constant $k_{p}$ for each $p \in P$, and let $\left\{x_{n}\right\}$ be a sequence in $X$. Then $\left\{x_{n}\right\}$ is a Cauchy sequence if and only if $d\left(x_{n}, x_{m}\right) \rightarrow 0$ as $n, m \rightarrow \infty$.

Definition 7. Let $S, G: X \rightarrow X$ be two single-valued function. We say that $S$ and $F$ are compatible if

$$
\lim _{n \rightarrow \infty} d\left(S F x_{n}, F S x_{n}\right)=0
$$

whenever $\left\{x_{n}\right\}$ is a sequence in $X$ such that $\lim _{n \rightarrow \infty} d\left(F x_{n}, S x_{n}\right)=0$.

In particular, $d(S F x, F S x)=0$ if $d(F x, S x)=0$ by taking $x_{n}=x$ for all $n$.

\section{Main results}

In this section, we assume that the function $\psi: C^{5} \rightarrow C, C(\subset E)$ is a proper closed convex and pointed "regular" cone with apex at the origin, satisfies the following conditions:

(i) $\psi$ is a strictly increasing, continuous function in each coordinate, and

(ii) for all $t \in C, \psi(t, t, t, 0,2 t)<t, \psi(t, t, t, 2 t, 0)<t$, and $\psi(t, 0,0, t, t)<t$.

Theorem 1. Let $(X, d)$ be a complete cone metric space, and let $S, T, F, G: X \rightarrow X$ be four mappings such that for all $x, y \in X$,

$$
d(S x, T y) \leq \psi(d(F x, G y), d(F x, S x), d(G y, T y), d(F x, T y), d(G y, S x)) .
$$

Assume that $T X \subset F X$ and $S X \subset G X$, and the pairs $\{S, F\}$ and $\{T, G\}$ are compatible. If $F$ or $G$ is continuous, then $S, T, F$ and $G$ have a unique common fixed point in $X$.

Proof. Given $x_{0} \in X$. Define the sequence $\left\{x_{n}\right\}$ recusively as follows:

$$
G x_{2 n+1}=S x_{2 n}=z_{2 n}, F x_{2 n+2}=T x_{2 n+1}=z_{2 n+1} .
$$

Since

$$
\begin{aligned}
d\left(z_{2 n}, z_{2 n+1}\right)= & d\left(S x_{2 n}, T x_{2 n+1}\right) \\
\leq & \psi\left(d\left(F x_{2 n}, G x_{2 n+1}\right), d\left(F x_{2 n}, S x_{2 n}\right), d\left(G x_{2 n+1}, T x_{2 n+1}\right), d\left(F x_{2 n}, T x_{2 n+1}\right),\right. \\
& \left.d\left(G x_{2 n+1}, S x_{2 n}\right)\right) \\
\leq & \psi\left(d\left(z_{2 n-1}, z_{2 n}\right), d\left(z_{2 n-1}, z_{2 n}\right), d\left(z_{2 n}, z_{2 n+1}\right), d\left(z_{2 n-1}, z_{2 n+1}\right), d\left(z_{2 n}, z_{2 n}\right)\right) \\
\quad \leq & \left.\psi\left(d\left(z_{2 n-1}, z_{2 n}\right), d\left(z_{2 n-1}, z_{2 n}\right), d\left(z_{2 n}, z_{2 n+1}\right), d\left(z_{2 n-1}, z_{2 n}\right)+d\left(z_{2 n}, z_{2 n+1}\right)\right), 0\right),
\end{aligned}
$$

hence

$$
d\left(z_{2 n}, z_{2 n+1}\right) \leq d\left(z_{2 n-1}, z_{2 n}\right) .
$$


Similary, since

$$
\begin{aligned}
& d\left(z_{2 n+1}, z_{2 n+2}\right)=d\left(S x_{2 n+2}, T x_{2 n+1}\right) \\
& \leq \psi\left(d\left(F x_{2 n+2}, G x_{2 n+1}\right), d\left(F x_{2 n+2}, S x_{2 n+2}\right), d\left(G x_{2 n+1}, T x_{2 n+1}\right),\right. \\
& \left.\quad d\left(F x_{2 n+2}, T x_{2 n+1}\right), d\left(G x_{2 n+1}, S x_{2 n}\right)\right) \\
& \leq \psi\left(d\left(z_{2 n+1}, z_{2 n}\right), d\left(z_{2 n+1}, z_{2 n+2}\right), d\left(z_{2 n}, z_{2 n+1}\right), 0, d\left(z_{2 n}, z_{2 n+2}\right)\right) \\
& \left.\leq \psi\left(d\left(z_{2 n+1}, z_{2 n}\right), d\left(z_{2 n+1}, z_{2 n+2}\right), d\left(z_{2 n}, z_{2 n+1}\right), 0, d\left(z_{2 n}, z_{2 n+1}\right)+d\left(z_{2 n+1}, z_{2 n+2}\right)\right)\right),
\end{aligned}
$$

hence

$$
d\left(z_{2 n+1}, z_{2 n+2}\right) \leq d\left(z_{2 n}, z_{2 n+1}\right) .
$$

Now, for each natural number $m$, if we denote $c_{m}=d\left(z_{m}, z_{m+1}\right)$, then $\left\{c_{m}\right\}$ is a decreasing sequence in $C$. Thus it must converges to some $c \in C$ with $c \geq 0$, since $C$ is regular.

If $c>0$, then by the above inequalities, we have

(1) if $m$ is even, then $c \leq c_{m+1} \leq \psi\left(c_{m}, c_{m}, c_{m}, 0,2 c_{m}\right)$. Passing to the limit, as $m \rightarrow \infty$, we have $c \leq c \leq \psi(c, c, c, 0,2 c)<c$, which is a contradiction.

(2) if $m$ is odd, then $c \leq c_{m+1} \leq \psi\left(c_{m}, c_{m}, c_{m}, 2 c_{m}, 0\right)$. Passing to the limit, as $m \rightarrow \infty$, we have $c \leq c \leq \psi(c, c, c, 2 c, 0)<c$, which is a contradiction.

Thus, $c=0$.

Next, we claim that the following result holds:

for each $\gamma>0$, there is $n_{0}(\gamma) \in N$ such that for all $m, n \geq n_{0}(\gamma)$,

$$
d\left(z_{m}, z_{n}\right)<\gamma
$$

We shall prove $(*)$ by contradiction. Suppose that $(*)$ is false. Then there exists some $\gamma>0$ such that for all $k \in N$, there are $m_{k}, n_{k} \in N$ with $m_{k}>n_{k} \geq k$ satisfying:

(i) $m_{k}$ is even and $n_{k}$ is odd, (see Yen [4])

(ii) $d\left(z_{m_{k}}, z_{n_{k}}\right) \geq \gamma$, and

(iii) $m_{k}$ is the smallest even number such that the conditions $(i),(i i)$ hold.

Since $c_{m} \searrow 0$, by $(i i)$, we have $\lim _{k \rightarrow \infty} d\left(z_{m_{k}}, z_{n_{k}}\right)=\gamma$, and

$$
\begin{aligned}
& \gamma \leq d\left(z_{m_{k}}, z_{n_{k}}\right)=d\left(S x_{m_{k}}, T x_{n_{k}}\right) \\
& \quad \leq \psi\left(d\left(F x_{m_{k}}, G x_{n_{k}}\right), d\left(F x_{m_{k}}, S x_{m_{k}}\right), d\left(G x_{n_{k}}, T x_{n_{k}}\right), d\left(F x_{m_{k}}, T x_{n_{k}}\right), d\left(G x_{n_{k}}, S x_{m_{k}}\right)\right) \\
& \quad \leq \psi\left(d\left(z_{m_{k}-1}, z_{n_{k}-1}\right), d\left(z_{m_{k}-1}, z_{m_{k}}\right), d\left(z_{n_{k}-1}, z_{n_{k}}\right), d\left(z_{m_{k}-1}, z_{n_{k}}\right), d\left(z_{n_{k}-1}, z_{m_{k}}\right)\right) \\
& \quad \leq \psi\left(c_{m_{k}-1}+d\left(z_{m_{k}}, z_{n_{k}}\right)+c_{n_{k}-1}, c_{m_{k}-1}, c_{n_{k}-1}, c_{m_{k}-1}+d\left(z_{m_{k}}, z_{n_{k}}\right), c_{n_{k}-1}+d\left(z_{m_{k}}, z_{n_{k}}\right)\right)
\end{aligned}
$$

Letting $k \rightarrow \infty$, we get $\gamma \leq \psi(\gamma, 0,0, \gamma, \gamma)<\gamma$, a contradiction. It follows from $(*)$ that the sequence $\left\{z_{n}\right\}$ must be a Cauchy sequence, hence $\left\{z_{n}\right\}$ converges to some $z \in X$. So, $F x_{2 n} \rightarrow z, G x_{2 n+1} \rightarrow z, S x_{2 n} \rightarrow z$ and $T x_{2 n+1} \rightarrow z$ as $n \rightarrow \infty$. 
Assume $F$ is continuous. Then we have $F^{2} x_{2 n} \rightarrow F z$ and $F S x_{2 n} \rightarrow F z$ as $n \rightarrow \infty$. Since $S$ and $F$ are compatible and $d\left(S x_{2 n}, F x_{2 n}\right) \rightarrow 0$ as $n \rightarrow \infty$, from

$$
d\left(S F x_{2 n}, F z\right) \leq d\left(S F x_{2 n}, F S x_{2 n}\right)+d\left(F S x_{2 n}, F z\right),
$$

we deduce that $d\left(S F x_{2 n}, F z\right) \rightarrow 0$ as $n \rightarrow \infty$.

For any $n \in N$,

$$
\begin{aligned}
d( & \left.S F x_{2 n}, T x_{2 n+1}\right) \\
\leq & \psi\left(d\left(F^{2} x_{2 n}, G x_{2 n+1}\right), d\left(F^{2} x_{2 n}, S F x_{2 n}\right), d\left(G x_{2 n+1}, T x_{2 n+1}\right), d\left(F^{2} x_{2 n}, T x_{2 n+1}\right),\right. \\
& \left.d\left(G x_{2 n+1}, S F x_{2 n}\right)\right), \text { and } \\
d\left(S z, T x_{2 n+1}\right) & \\
\leq & \psi\left(d\left(F z, G x_{2 n+1}\right), d(F z, S z), d\left(G x_{2 n+1}, T x_{2 n+1}\right), d\left(F z, T x_{2 n+1}\right),\right. \\
& \left.d\left(G x_{2 n+1}, S z\right)\right) .
\end{aligned}
$$

As $n \rightarrow \infty$, we get

$$
\begin{aligned}
& d(F z, z) \leq \psi(d(F z, z), 0,0, d(F z, z), d(F z, z)), \text { and } \\
& d(S z, z) \leq \psi(d(F z, z), d(F z, S z), 0, d(F z, z), d(z, S z)),
\end{aligned}
$$

which imply that $F z=z$ and $S z=z$.

Select $z^{\prime} \in X$ such that $G z^{\prime}=z=S z$. Then $T G z^{\prime}=T z$, and

$$
\begin{aligned}
& d\left(z, T z^{\prime}\right)=d\left(S z, T z^{\prime}\right) \\
& \quad \leq \psi\left(d\left(F z, G z^{\prime}\right), d(F z, S z), d\left(G z^{\prime}, T z^{\prime}\right), d\left(F z, T z^{\prime}\right), d\left(G z^{\prime}, S z\right)\right) \\
& \quad \leq \psi\left(0,0, d\left(z, T z^{\prime}\right), d\left(z, T z^{\prime}\right), 0\right)
\end{aligned}
$$

which implies that $T z^{\prime}=z$ and so $G T z^{\prime}=G z$. Since $T$ and $G$ are compatible and $d\left(T z^{\prime}, G z^{\prime}\right)=0$, we get $d(T z, G z)=d\left(T G z^{\prime}, G T z^{\prime}\right)=0$, which implies $T z=G z$. Since

$$
\begin{aligned}
& d(z, T z)=d(S z, T z) \\
& \quad \leq \psi(d(F z, G z), d(F z, S z), d(G z, T z), d(F z, T z), d(G z, S z)) \\
& \quad \leq \psi(d(z, T z), 0,0, d(z, T z), d(z, T(z))),
\end{aligned}
$$

we have $d(z, T z)=0$, and so $T z=z$.

Hence $z$ is a common fixed point of $S, T, F$ and $G$ with $S z=T z=z=F z=G z$.

Let $y$ be a common fixed point of $S, T, F$ and $G$. Since

$$
\begin{aligned}
& d(y, z)=d(S y, T z) \\
& \quad \leq \psi(d(F y, G z), d(F y, S y), d(G z, T z), d(F y, T z), d(G z, S y)) \\
& \quad=\psi(d(y, z), 0,0, d(y, z), d(y, z))
\end{aligned}
$$

This implies $y=z$. Hence $z$ is the unique common fixed point of $S, T, F$ and $G$. 
The proof for $G$ continuous is similar.

\section{Acknowledgements}

The author would like to thank the referees for their valuable suggestions which improved the presentation of the paper.

\section{References}

[1] L. G. Huang, X. Zhang, Cone metric spaces and fixed point theorems of contractive mappings, J. Math. Anal. appl.322(2007), 1468-1476.

[2] Y. C. Lin, On Generalized Vector Equilibrium Problems, Nonlinear Anal.In Press(2008).

[3] P. Vetro, Common fixed points in cone metric spaces, Rend. Circ. Mat. Palermo.56(2007), 464-468.

[4] C. L. Yen, On common fixed points II, Tamkang J. Math., Tamkang College of Art and Sci.4(1973), 57-60.

Department of Applied Mathematics, National Hsinchu, University of Education, Taiwan.

E-mail: ming@mail.nhcue.edu.tw

Department of Applied Mathematics, National Hsinchu, University of Education, Taiwan.

E-mail: thchang@mail.nhcue.edu.tw 